\title{
Optimized Screw Trajectory for Lumbar Cortical Bone Trajectory Pedicle Screws Based on Clinical Outcome: Evidence Favoring the Buttress Effect Theory
}

\author{
Shunji Asamoto ${ }^{1, *}$ Kota Kojima ${ }^{2, *}$ Michael Winking ${ }^{3}$ Andreas Jödicke ${ }^{4}$ Masayuki Ishikawa ${ }^{5}$
} Shinichi Ishihara ${ }^{5}$ Wolfgang Deinsberger ${ }^{6}$ Jun Muto ${ }^{1}$ Makoto Nishiyama ${ }^{5}$

\footnotetext{
${ }^{1}$ Department of Neurosurgery, International University of Health and Welfare, Tokyo, Japan

${ }^{2}$ Department of Orthopaedic Surgery, Keio University School of Medicine, Shinjuku, Japan

${ }^{3}$ ZW-O Spine Center Osnabrück, Osnabrueck, Germany

${ }^{4}$ Department of Neurosurgery, Vivantes Klinikum Neukolln Klinik fur Innere Medizin Pneumologie und Infektiologie, Berlin, Germany

${ }^{5}$ Spine and Spinal Cord Center, International University of Health and Welfare, Tokyo, Japan

${ }^{6}$ Department of Neurochirurgie, Klinikum Kassel, Kassel, Germany
}

\author{
Address for correspondence Kota Kojima, MBBS, Department of \\ Orthopaedic Surgery, Keio University School of Medicine, \\ 35 Shinanomachi, Shinjuku, Shinjuku 160-0016, Japan \\ (e-mail: dr.kota.kojima@keio.jp).
}

J Neurol Surg A 2018;79:464-470.

\begin{abstract}
\section{Keywords}

- posterior lumber fusion

- cortical bone trajectory

- buttress theory

- surgical technique

Background Cortical bone trajectory (CBT) is a relatively new technique for pedicle screw insertion in the field of spine surgery. Previous studies have demonstrated the significantly better pullout and toggle characteristics the new method offers, and it appears to have certain advantages over the widely used traditional trajectory. The mechanism of the pullout and toggle characteristics still remains unknown.

Purpose To report the medium- to long-term follow-up findings of patients who underwent posterior lumbar interbody fusion or posterior lumbar fusion (PLIF/PLF) at our institution and to discuss the ideal screw trajectory when using this technique.

Study Design Retrospective radiologic study.

Methods Fifty-five patients who underwent PLIF/PLF for lumbar spondylosis using the new technique between January 2011 and January 2016 were included. Clinical outcome was assessed using the Japanese Orthopaedic Association scores for low back pain and visual analog scale scores. Screw loosening was evaluated via the presence of a translucent zone surrounding the pedicle screw using plain X-radiography (Xp). The screw trajectory was evaluated by measuring the rostral range (RR) and the lateral range score (LRS). The RR is the angle between the line drawn along the distal end plate of the vertebra and the line drawn along the screw on the lateral view. The LRS is the score given depending on the position of the tip of the screw seen on the Xp taken in the anteroposterior (AP) view. The pedicle ring is equally divided into the medial third (zone $A$ ), central third (zone $B$ ), and the lateral third (zone $C$ ) by four vertical lines. A score of 0 to 3 points is given depending on the position of the tip of the screw: 0 , outside the pedicle ring; 1 , zone $A ; 2$, zone $B$; and 3 , zone $C$.

Results Bone fusion was recorded in 49 patients ( $49 / 55$ patients). The total number of screws with a visible translucent zone on Xp was 26 (26/242 screws). When the screws
\end{abstract}

The authors contributed equally.

received

May 8, 2016

accepted after revision

June 6, 2017

published online

April 30, 2018 (c) 2018 Georg Thieme Verlag KG

Stuttgart · New York
DOI https://doi.org/

$10.1055 / \mathrm{s}-0038-1641147$. ISSN 2193-6315. 
are inserted at a more acute angle in the lateral view (low RR) or when the screws are directed more medially (low LRS), the risk of developing a translucent zone around the pedicle screw increased.

Conclusion With CBT, the pedicle screws are inserted from the pars interarticularis that is rich in cortical bone, strong enough to withstand the substantial forces usually encountered in the region. Our clinical data suggest that the optimal screw trajectory is one where the screw is inserted at an angle $>22.55$ degrees (high RR) on the lateral view Xp and where the tip of the screw is directed toward the lateral third of the pedicle on the AP Xp. We believe the naturally occurring buttress effect that exists in the vertebra may give CBT a mechanical and anatomical advantage over the traditional screw trajectory.

\section{Introduction}

With the introduction of cortical bone trajectory (CBT), alternative techniques for lumbar pedicle screw insertion have become a topic of interest in recent years. Santoni et al ${ }^{1}$ demonstrated the good pullout and toggle characteristics the CBT technique offers, and because the screws are inserted in a mediolateral trajectory, it is considered less invasive compared with the traditional transpedicular method. However, there is still little consensus regarding the optimal screw insertion angle when using CBT. As a result, there are no intra- and postoperative criteria available for surgeons to assess the screw position.

In our institution, we have performed $>100$ cases of posterior spinal fusion using the CBT, and the results have been highly promising so far. This article reports our medium- to long-term follow-up findings and discusses the ideal screw trajectory when using this technique.

\section{Materials and Methods}

A total of 104 patients who underwent posterior lumbar interbody fusion or posterior lumbar fusion (PLIF/PLF) for lumbar spondylosis using the CBT technique between January 2011 and January 2016 were included. Exclusion criteria were patients followed $<1$ year, patients with isthmic spondylosis, and patients who required more than three interbody fusions.

Clinical outcome was assessed using the Japanese Orthopaedic Association (JOA) score for low back pain ${ }^{2,3}$ (- Table 1) and visual analog scale (VAS) scores. Screw loosening was evaluated via the presence of a translucent zone surrounding the pedicle screw using plain X-radiography (Xp).

The screw trajectory was evaluated by measuring the rostral range (RR) and the lateral range score (LRS). The RR is the angle between the line drawn along the distal end plate of the vertebra and the line drawn along the inserted screw seen on the lateral view (-Fig. 1). The LRS is a score given depending on the position of the tip of the screw seen on the $\mathrm{Xp}$ taken in the anteroposterior (AP) view with the vertebra parallel to the axis. The pedicle ring is equally divided into the medial third (zone A), central third (zone B), and the lateral third (zone $C$ ) by four vertical lines that are parallel to the vertebral axis. A score of 0 to 3 points is given depending on the position of the tip of the screw: 0 , outside the pedicle ring; 1 , zone A; 2, zone B; and 3, zone C (-Fig. 2).

All of our pedicle screws were polyaxial, and their dimensions were selected preoperatively based on the patient's computed tomography (CT) scans and intraoperatively using standard probing techniques.

\section{Statistical Methods}

Values are expressed as the mean plus or minus the standard error of the mean. Differences in baseline characteristics were tested using the Student $t$ test for continuous variables and the chi-square test for categorical variables. Nonnormally distributed variables were compared using the Mann-Whitney nonparametric $U$ test. A $p<0.05$ was considered statistically significant. Cut-off values were derived using Youden's index. We used SPSS statistical software, v.22 (IBM Corp., Armonk, New York, United States) for all analyses.

\section{Results}

The final number of patients included in this study was 55 (after omitting patients who fit the exclusion criteria). The mean age of the patients was 66.3 years (range: $21-89$ years), there were 28 male patients (age range: $39-89$; mean age: 68 ) and 27 female patients (age range: 21-81; mean age: 67), respectively. The mean follow-up period was 30.5 months (range: 12-60 months). The total number of screws analyzed was 242 , and the number of screws inserted into each vertebra were as follows: L2, 4; L3, 45; L4, 102 ; and L5, 91 screws.

The mean JOA score preoperatively was $13.9 \pm 0.6$ (range: 3-24), and the mean JOA score postoperatively was $27.6 \pm 1.9$ (range: 21-29). The mean VAS score preoperatively was $8.6 \pm 0.1$ (range: $5.5-10$ ), and the mean VAS score postoperatively was $0.4 \pm 0.1$ (range: $0-5$ ). All 55 patients had an improved JOA and VAS score on their most recent postoperative follow-up. The mean RR was $28.7 \pm 0.44$ degrees (range: 7.0-48.3), and the mean LRS was $2.76 \pm 0.04$ (range: $0-3$ ). Two screws scored 0 on the LRS (2/242 [0.8\%]).

Bone fusion was recorded in 49 patients (49/55 patients [89.1\%]). The total number of screws with a visible transluscent zone on Xp was 26 (26/242 screws [10.8\%]). One patient developed an adjacent segment disease (1/55 patients [1.8\%]). We identified at least one pedicle screw with a 
Table 1 Japanese Orthopaedic Association score for low back pain

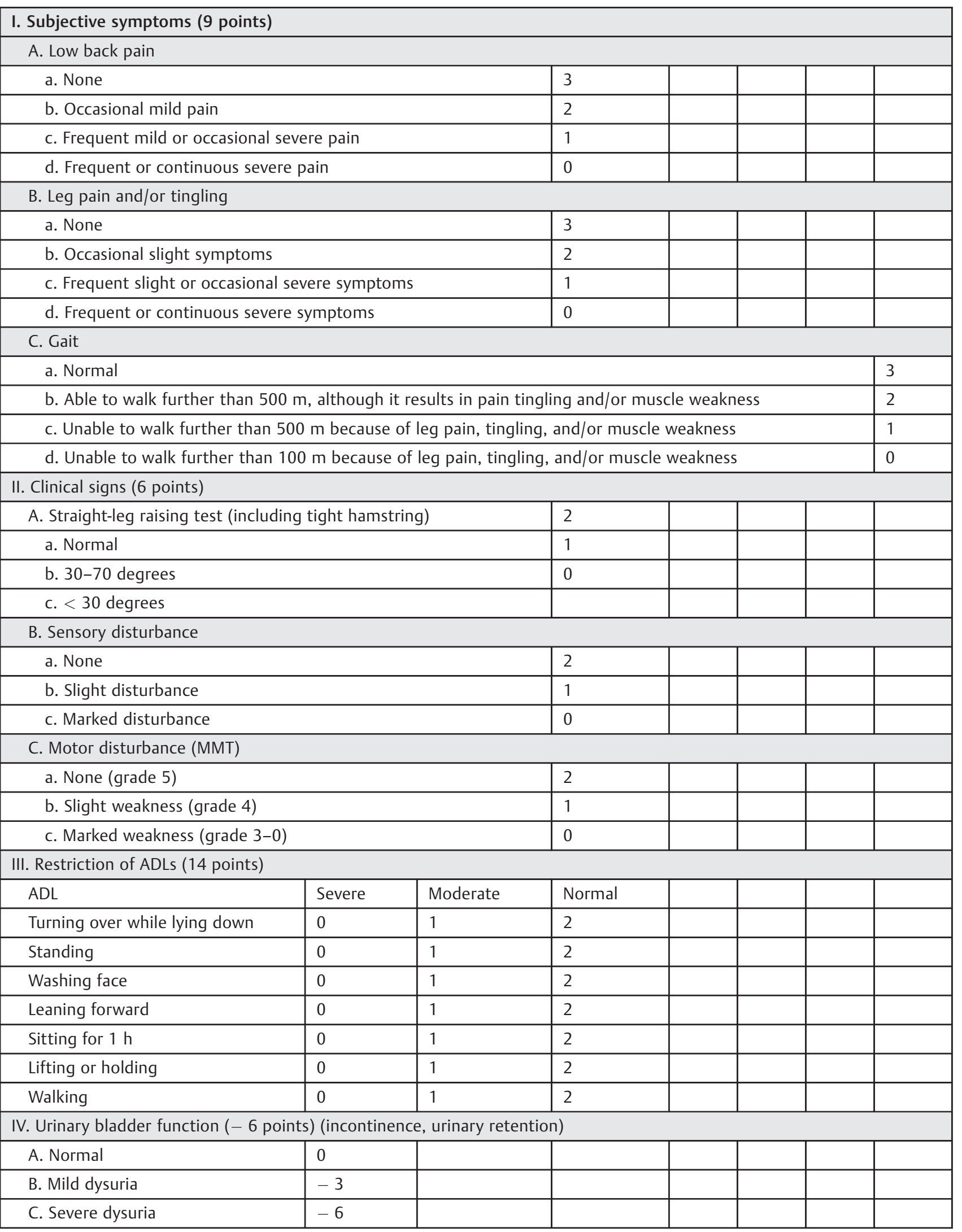

Abbreviations: ADL, activity of daily living; MMT, manual muscle testing. 


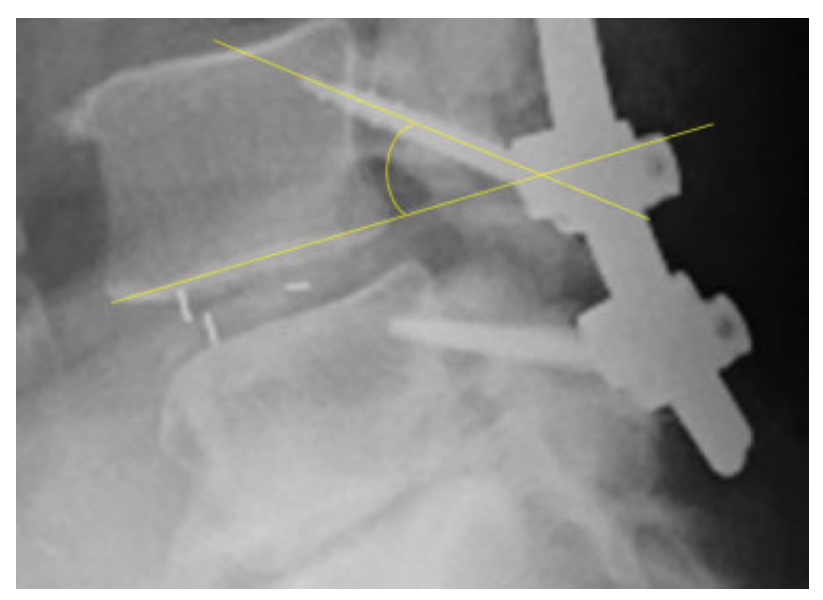

Fig. 1 Rostral range: the angle between a line drawn along the distal end plate of the vertebra and a line drawn along the inserted screw seen on the plain X-radiograph in the lateral view.

translucent zone in all six patients with poor bone fusion. Surgical revision rate was zero, and the screw pullout rate was zero.

A significant difference was noted in the mean JOA score improvement between patients without a visible translucent zone on Xp (14.4 \pm 0.7 points) and patients with a visible translucent zone (11.6 \pm 1.3 points) ( $p<0.05$; - Table 2$)$.

There was no significant difference in the mean VAS score improvement between patients without a visible translucent zone on Xp $(8.2 \pm 0.2 \mathrm{~mm})$ and patients with a visible translucent zone $(8.1 \pm 0.4 \mathrm{~mm})(\mathrm{p}>0.05$; - Table 2$)$.

There was a significant difference between the mean RR between patients without a visible translucent zone on Xp (30.0 \pm 0.4 degrees) and patients with a visible translucent zone (17.2 \pm 1.3 degrees) ( $p<0.01$; - Table 3 ). The angle at which the Youden's index ( $\mathrm{J}$ ) reached its highest value was 22.55 degrees (J: 0.8; area under the curve: 0.93).

There was a significant difference between the mean LRS between patients without a visible translucent zone on Xp
$(2.9 \pm 0.02)$ and patients with a visible translucent zone $(1.6 \pm 0.14)(p<0.01 ;$ - Table 3$)$.

\section{Discussion}

Using CBT over the traditional trajectory has several advantages. The screw insertion point is more medial, meaning the required muscle dissection is much less and, in general, the intraoperative blood loss is reduced. The screws are pointed away from the nerve root so the risks of neurovascular injury are lower. ${ }^{4}$ There is potentially a higher risk of pedicle fractures; thus preoperative and intraoperative assessment is essential.

In this study, we analyzed the medium- to long-term outcome of patients following PLF/PLIF using CBT both from a clinical and a radiologic standpoint. All patients showed improvement in their JOA and VAS scores at their most recent postoperative follow-up, regardless of the presence of a translucent zone on their Xp. However, it appears that patients without a visible translucent zone on their Xp have a greater improvement in their JOA score compared with those with a translucent zone seen around the pedicle screws. The one patient with adjacent segment disease is currently being closely observed, but the patient's symptoms have not warranted additional surgery so far.

We previously reported a study comparing the pedicle screw and cortical bone contact between the traditional trajectory and CBT using CT to elucidate the reason why, despite using smaller and shorter screws in CBT, the pullout and toggle resistance are comparable between the two trajectories. $^{5}$ In this study we concluded that when the pedicle screws are inserted using CBT starting at the pars interarticularis, the screw penetrates a region that is far richer in cortical bone compared with using the traditional trajectory, which must be contributing to the pullout and toggle characteristics.

The results from this present study suggest that when the screws are inserted at a more acute angle in the lateral view (low RR) or when the screws are directed more medially (low LRS), the risk of developing a translucent zone around the pedicle screw increases. Based on the Youden's index

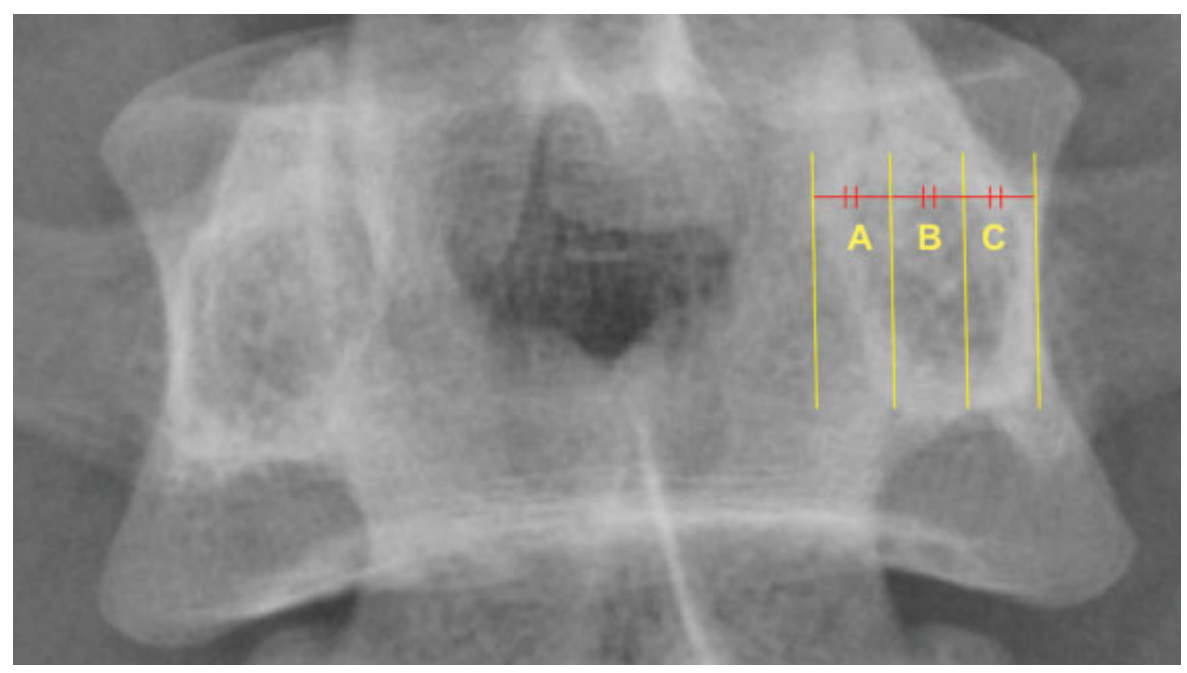

Fig. 2 Lateral range score: score assigned depending on the position of the tip of the screw seen on X-radiography taken in the anteroposterior view with the vertebra parallel to the axis. The pedicle ring is equally divided into the medial third (zone A), central third (zone B), and the lateral third (zone C) by four vertical lines that are parallel to the vertebral axis. A score of 0 to 3 is given depending on the position of the tip of the screw. 
Table 2 Japanese Orthopaedic Association score versus visual analog scale of patients before and after surgery ${ }^{\mathrm{a}}$

\begin{tabular}{|l|l|l|l|l|}
\hline \multirow{2}{*}{} & \multicolumn{2}{|l|}{ JOA } & VAS \\
\cline { 2 - 5 } & Preoperative & Postoperative & Preoperative & Postoperative \\
\hline $\begin{array}{l}\text { All patients } \\
(n=55)\end{array}$ & $13.9( \pm 0.6)$ & $27.6( \pm 1.9)$ & $8.6( \pm 0.1)$ & $0.4( \pm 0.1)$ \\
\hline & JOA & Postoperative & Preoperative & Postoperative \\
\cline { 2 - 5 } & Preoperative & $28.0( \pm 0.3)$ & $8.6( \pm 0.2)$ & $0.3( \pm 0.1)$ \\
\hline $\begin{array}{l}\text { translucent zone }(-) \\
(n=39)\end{array}$ & $13.5( \pm 0.7)$ & $26.5( \pm 0.6)$ & $8.8( \pm 0.1)$ & $0.6( \pm 0.4)$ \\
\hline $\begin{array}{l}\text { translucent zone }(+) \\
(n=16)\end{array}$ & $15.1( \pm 1.2)$ & & 0.45 & \\
\hline$p$ value & 0.23 & VAS improvement & & \\
\hline & JOA improvement & $8.2( \pm 0.2)$ & & \\
\hline $\begin{array}{l}\text { translucent zone }(-) \\
(n=39)\end{array}$ & $14.4( \pm 0.7)$ & $8.1( \pm 0.4)$ & & \\
\hline $\begin{array}{l}\text { translucent zone }(+) \\
(n=16)\end{array}$ & $11.6( \pm 1.3)$ & 0.79 & & \\
\hline$p$ value & 0.04 & & \\
\hline
\end{tabular}

Abbreviations: JOA, Japanese Orthopaedic Association; VAS, visual analog scale.

${ }^{a}$ There was a significant difference between the mean JOA score improvement between patients without a visible translucent zone on X-radiography and patients with a visible translucent zone.

Table 3 Rostral range versus lateral range score of the inserted pedicle screws with and without a visible translucent zone on X-radiography ${ }^{\text {a }}$

\begin{tabular}{|l|l|l|}
\hline & RR, degrees & LRS \\
\hline $\begin{array}{l}\text { All screws } \\
(n=242)\end{array}$ & $28.67( \pm 0.44)$ & $2.76( \pm 0.04)$ \\
\hline & RR, degrees & LRS \\
\hline $\begin{array}{l}\text { Translucent zone } \\
(-)(n=216)\end{array}$ & $30.0( \pm 0.4)$ & $2.9( \pm 0.02)$ \\
\hline $\begin{array}{l}\text { Translucent zone } \\
(+)(n=26)\end{array}$ & $17.2( \pm 1.3)$ & $1.6( \pm 0.14)$ \\
\hline$p$ value & $<0.01$ & $<0.01$ \\
\hline
\end{tabular}

Abbreviations: LRS, lateral range score; RR, rostral range.

aThere was a significant difference between the mean RR and LRS between patients without a visible translucent zone and patients with a visible translucent zone.
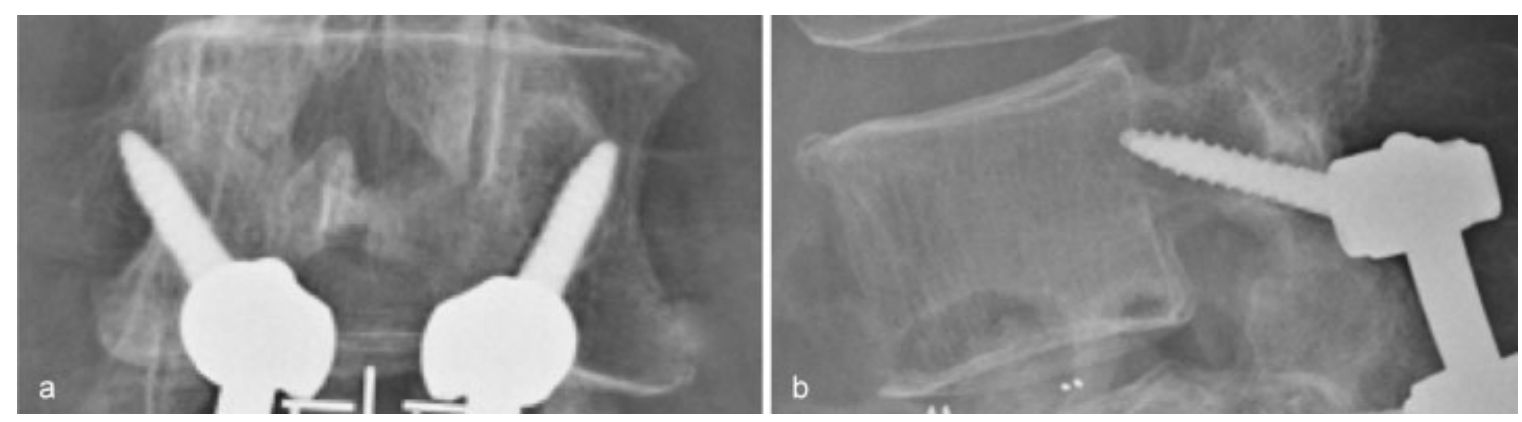

Fig. 3 Method to determine the optimal screw insertion based on our clinical findings: (a) High angle of insertion (rostral range) on the lateral view. (b) Pedicle screw tip placed in the lateral third of the pedicle (zone C). calculation, the cut-off value for the RR from our data is 22.55 degrees. Therefore, we believe the ideal screw trajectory when using $\mathrm{CBT}$ begins at the pars interarticularis with the pedicle screw inserted at an angle $>22.55$ degrees (high $\mathrm{RR}$ ) on the lateral view Xp and with the tip of the screw resting on the lateral third of the pedicle (zone $\mathrm{C}$ ) on the AP view Xp. It is unclear whether the presence of a translucent zone causes the slight difference in JOA improvement, but we feel it deserves further investigation.

In our institution, we currently use a C-arm intraoperatively to achieve the optimal screw angle during screw insertion. Starting at the pars interarticularis, on the lateral view, we direct the screw so the angle of insertion is as high as possible without it breaching the pedicle (high RR). On the AP view, we adjust the $C$-arm so the vertebral body is parallel to the line of view and insert the screw so the tip of the screw sits on the lateral third (zone C, high LRS) of the pedicle (-Fig. 3). It is essential that the surgeon studies the patient's 


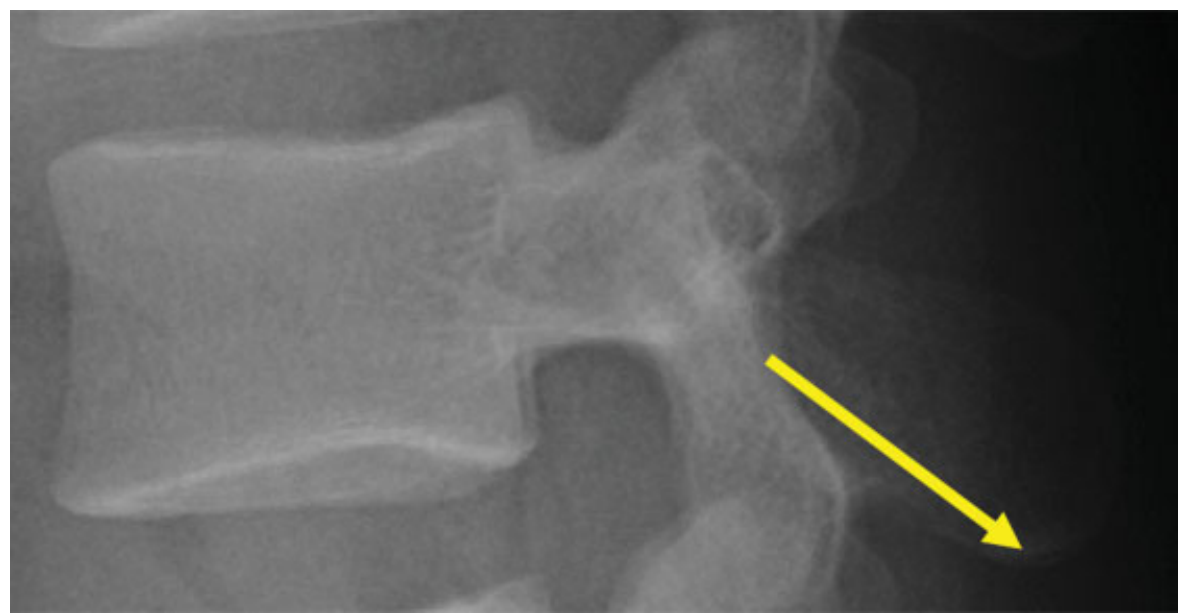

Fig. 4 Vector of forces (resultant force) acting on the pars interarticularis (arrow).

CT images before surgery to minimize the risk of the screw breaching the pedicle.

Although much is still to be elucidated before fully understanding our results, previous studies have given us some insight. In 2009, Santoni et al demonstrated the significant anchoring property of the CBT when performing posterior lumbar spine fusion. A human cadaveric biomechanical
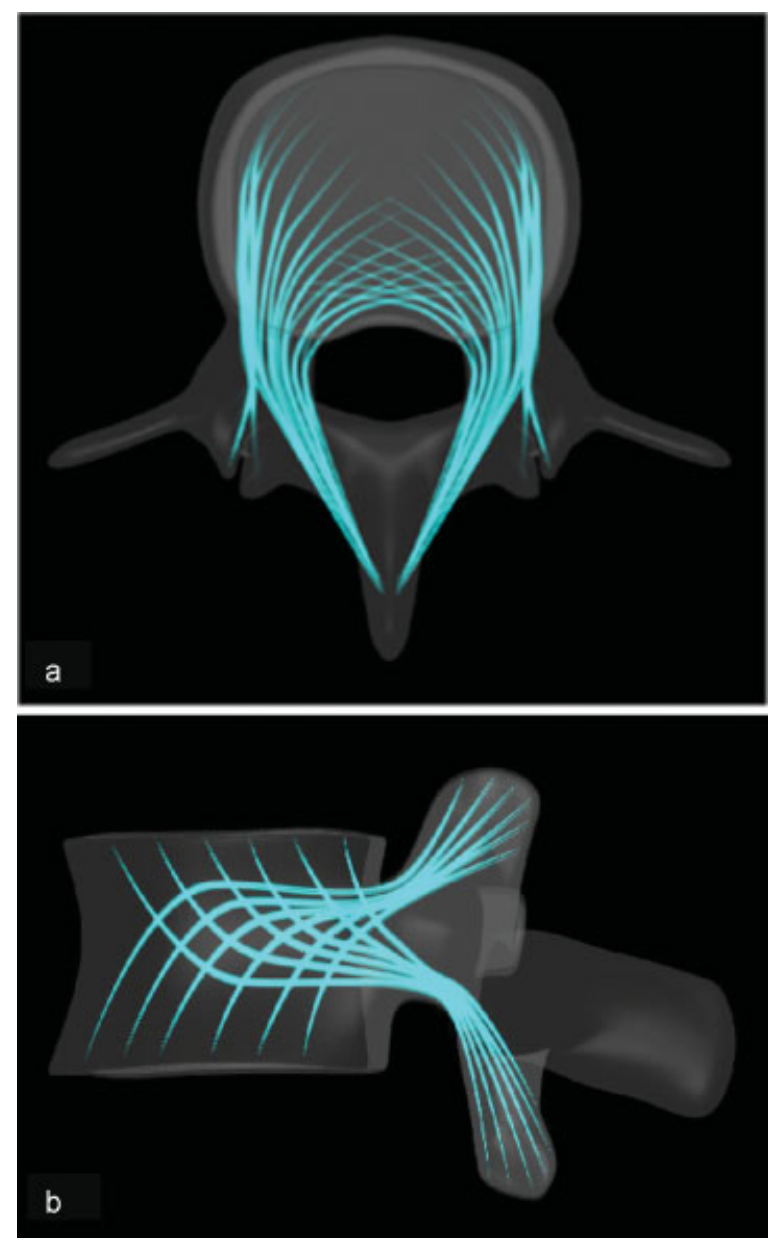

Fig. 5 Cortical bone trabeculation within the lumbar vertebra. (a) Craniocaudal view. (b) Lateral view. study was conducted to evaluate the mechanical properties of the CBT compared with the traditional trajectory. Two pedicle screws were inserted into a human vertebra with one side using the traditional transpedicular path and the other using the new CBT. Pullout and toggle testing was performed,
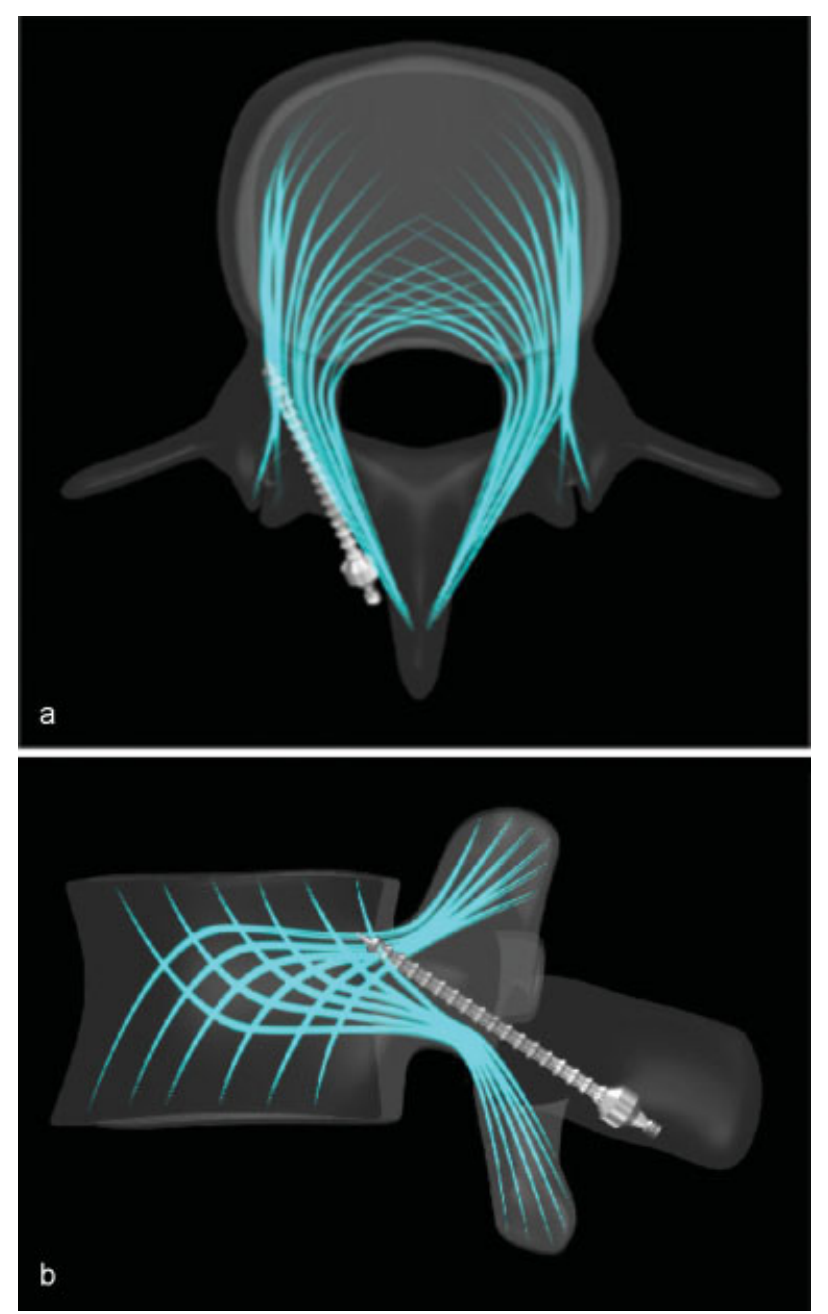

Fig. 6 (a) Craniocaudal view and (b) lateral view of a lumbar vertebra with a pedicle screw positioned along the cortical bone trabeculation. 
and the results showed that the screws inserted using CBT had equivalent pullout and toggle characteristics compared with those using the traditional trajectory. ${ }^{1}$

In 1976, Troup found that spondylolisthesis and spondylolysis commonly occur at the pars interarticularis (isthmus). ${ }^{6}$ The article focuses on the direction and magnitude of stress the pars interarticularis has to withstand with various lumbar movements (-Fig. 4). It describes the two layers of thick cortical bone that exist in the region and hypothesizes that it is contributing to the strength of the pars interarticularis.

Gallois and Japiot were the first to report the cortical bone trabeculation within the lumbar vertebrae by illustrating the flow of cortical bone that starts from the body, through the pedicles to the posterior structures such as the lamina and articular process. ${ }^{7}$ According to their report, the trabecular structure appears to start from the vertebral body and extends toward the lamina and superior/inferior articular processes. In the vertical plane, the trabeculae begin at the superior and inferior aspect of the vertebral body and flow toward the inferior and superior articular processes, respectively. In the horizontal plane, the trabeculae start at the anterior aspect of the vertebral body and flow toward the lamina and the transverse processes (-Fig. 5).

We believe there is an added buttress effect when pedicle screws are inserted along the flow of cortical bone trabeculation described by Gallois and Japiot. This will result in added protection, which may explain why the screws inserted with a higher LRS (i.e., screws directed toward the lateral wall of the pedicle) had a lower rate of translucent zone formation. The fact that the screw direction in the new trajectory matches the vector of forces acting on the pars interarticularis may also play a part (-Fig. 6).

\section{Conclusion}

With CBT, the pedicle screw is inserted from the pars interarticularis that is rich in cortical bone $e^{5,8,9}$ to withstand the forces encountered from the various lumbar movements, and it is directed along the natural trabecular flow that exists in the lumbar vertebra. We believe the naturally occurring buttress effect that exists in the vertebra may provide CBT a mechanical and anatomical advantage over the traditional trajectory ("The Buttress Effect Theory," a paper presented at the 2013 annual meeting of the Japanese Society of Spinal Surgery, Nagoya, Japan). Our clinical data suggest that the optimal screw trajectory for CBT is one where the pedicle screw is inserted at an angle $>22.55$ degrees (high RR) on the lateral view Xp and where the tip of the pedicle screw is resting on the lateral third of the pedicle (zone $\mathrm{C}$ ) on the AP view Xp.

\section{References}

1 Santoni BG, Hynes RA, McGilvray KC, et al. Cortical bone trajectory for lumbar pedicle screws. Spine J 2009;9(05):366-373

2 Fukui M, Chiba K, Kawakami M, et al; Subcommittee of the Clinical Outcome Committee of the Japanese Orthopaedic Association on Low Back Pain and Cervical Myelopathy Evaluation. JOA Back Pain Evaluation Questionnaire (JOABPEQ)/JOA Cervical Myelopathy Evaluation Questionnaire (JOACMEQ). The report on the development of revised versions. April 16, 2007. J Orthop Sci 2009;14 (03):348-365

3 Izumida S, Inoue S. Assessment of treatment for low back pain [in Japanese]. J Jpn Orthop Assoc 1986;60:391-394

4 Phan K, Hogan J, Maharaj M, Mobbs RJ. Cortical bone trajectory for lumbar pedicle screw placement: a review of published reports. Orthop Surg 2015;7(03):213-221

5 Kojima K, Asamoto S, Kobayashi Y, Ishikawa M, Fukui Y. Cortical bone trajectory and traditional trajectory-a radiological evaluation of screw-bone contact. Acta Neurochir (Wien) 2015;157(07): 1173-1178

6 Troup JDG. Mechanical factors in spondylolisthesis and spondylolysis. Clin Orthop Relat Res 1976;(117):59-67

7 Gallois M, Japiot M. Architecture intérieure des vertèbres (statique et physiologie de la colonne vertébrale). Rev Chir 1925;63:687-708

8 Cyron BM, Hutton WC. Variations in the amount and distribution of cortical bone across the partes interarticulares of L5. A predisposing factor in spondylolysis? Spine 1979;4(02):163-167

9 Krenz J, Troup JDG. The structure of the pars interarticularis of the lower lumbar vertebrae and its relation to the etiology of spondylolysis, with a report of a healing fracture in the neural arch of a fourth lumbar vertebra. J Bone Joint Surg Br 1973;55(04):735-741 